\title{
Examining fallacies in diagnostic reasoning
}

\author{
C. W. van Staden MBChB MMed(Psych) MD FCPsych(SA) FTCL UPLM
}

Professor of Philosophy \& Psychiatry, Department of Psychiatry, University of Pretoria, Pretoria, South Africa

The paper by Gurova takes issue with the claim of Meehl that 'understanding makes it normal' is a fallacy in diagnostic reasoning [1,2]. Her paper prompts the more general question of appropriate methods for testing whether a specific way of reasoning in making a clinical diagnosis is fallacious. So prompted, I will compare the methods that the Gurova paper deploys with some of requirements for testing whether a particular way of reasoning is fallacious. It should become clear in this comparison that the dictum 'understanding it makes it normal' per se is insufficient to proclaim whether reasoning by it is fallacious.

For purposes of matching methods of examining a potential fallacy with those deployed in Gurova's paper, let us first consider the argument of the Gurova paper. It argues that the attribution of fallacy status to this way of reasoning is undermined, if not refuted, when one considers that this way of reasoning (i) is commonly used as supported by empirical evidence; (ii) has a potential cultural nature/origin; and (iii) is used in ways that vary independently of professional experience.

Furthermore, the fallacy status of this way of reasoning is undermined if (iv) a commitment to the mere physiological causal validity of a diagnosis is relinquished; and (v) one realizes that understanding a condition as being normal (physiologically) does not preclude further utilities such as further care or abnormal consequences.

The first three (i-iii earlier) of the considerations that undermine the fallacy idea are all based on empirical findings. So one may ask how empirical findings may render reasoning fallacious (or not). In one way, empirical findings do not have such reach. That is, the logical inferential validity of reasoning should be independent of empirical findings $[3,4]$. One reason for this requirement is that just because an inference is commonly made, that serves as no guarantee of validity. On the contrary, some fallacies are inferred rather commonly [5]. Applied to the Gurova paper, that means that the empirically based considerations (i) to (iii) neither guarantee the validity of the inference by which it is concluded that 'under-standing it makes it normal', nor do they guarantee the validity of the inference by which it is concluded that 'understanding it makes it normal' is fallacious.

Other than for the logical inferential validity, however, empirical findings do have a place in examining ways of reasoning. That is, when examining a premise that is an empirical matter. If the premise in the reasoning is 'John Smith was ill', the truth of that depends on the empirical evidence as to whether John was actually ill. But for the premise 'a chair is a piece of furniture', no number of empirical observations could support or refute the claim. Applied to the Gurova paper, we need to consider whether 'under-standing it makes it normal' is an empirical claim, as well as whether the claim " "Understanding it makes it normal" is fallacious' is an empirical claim. Are these claims similar to 'John Smith was ill' or 'a chair is a piece of furniture'. Inasmuch as they are similar to the former, empirical findings are relevant. Inasmuch as these claims are similar to 'a chair is a piece of furniture', empirical findings are irrelevant. The extent to which these claims are empirical seems 
rather uncertain to me, but what should be clear is that they are certainly conceptual claims, no less so for the meaning embedded in the dictum as well as in the concept of a fallacy.

As a conceptual matter then, the Gurova paper follows a suitable method insofar as it examines the conceptual underpinnings of 'understanding it makes it normal' as well as its fallacy attribution. So it describes a conceptual underpinning of the fallacy attribution to the dictum as that of a commitment to the mere physiological causal validity of a diagnosis (see iv earlier). The fallacy attribution boils down to more or less this: only physiologically caused conditions are valid and being so caused, they are present and by nature abnormal in a particular case independent of 'understanding'. Thus, 'understanding' should have no bearing on the presence of the abnormal condition being present.

One could take issue with this underpinning commitment in various ways: one can argue against the respective ideas that (i) all abnormality is caused physiologically; (ii) that validity of a diagnosis depends necessarily on (the presumption of) physiological causes; (iii) that validity of a diagnosis depends necessarily on physiological causes being known; (iv) physiologically caused conditions are necessarily abnormal; and ( $v$ ) physiologically caused conditions would be immune to 'understanding'. One could also draw similarities between this narrow understanding of a valid diagnosis and that of the pathological thinking in somato-form disorders [6]. The Gurova paper does not take issue in any of these ways, and argues that even if committed to the idea that only physiologically caused conditions are valid independent of 'understanding', psychiatric conditions are not constrained by this validity requirement, which means they may accommodate the contextual influence of 'understanding' in the diagnostic reasoning without its being fallacious.

The Gurova paper's further conceptual challenge (see v earlier) to the fallacy attribution is linked to the former issue of physiological causal validity. The paper cites Kendell and Jablensky [7], saying instead of this validity requirement, a utility requirement is more applicable to psychiatric conditions. If so accepted, the Gurova paper argues that a utility requirement instead of this validity requirement allows for the contextual influence of 'under-standing' in diagnostic reasoning.

Notwithstanding the merits of the Gerova paper regarding these conceptual underpinnings (numbered iv and v earlier), a critical aspect about the debate should be observed. The critical aspect is this: the dictum 'understanding it makes it normal' per se is insufficient for judging whether it is expressing fallacious diagnostic reasoning. But what else is required then?

This question brings us back to considering appropriate methods for testing whether a specific way of reasoning in making a clinical diagnosis is fallacious. In examining reasoning, the traditional aspects for consideration are the truth value of the premises in the argument, the logical inference, the contextual and conceptual congruence among the premises and the conclusion, and the cogency of the conclusion [3-5].

So far, we have considered the validity of logical inferences, which is independent of empirical findings. Applied to the diagnostic reasoning by the dictum 'understanding it makes it normal', the premises from which this inference is made need to be known before the validity of the inference can be examined. In this regard, empirical work may indeed contribute by making known what the premises are. Gurova's references to empirical work may suitably represent a step in this direction. 
Knowing the premises would also allow for examining their individual truth values, the conceptual and contextual congruencies between them and between them and the conclusion. Without these, we may only consider the conclusion, in this case 'under-standing it makes it normal', and try to unpack what the entailed premises potentially are - that is, what the embedded meanings may be.

The embedded meanings are also critically important in examining contextual and conceptual congruence. The lack of contextual or conceptual congruence may invite fallacies of equivocation. One may easily spot where fallacies of equivocation may arise considering the highly ambiguous dictum 'understanding it makes it normal'. The indefinite pronoun 'it' needs to indicate the very same thing at both places in the dictum. That is, the 'it' that is understood should be the very thing that is considered normal. Now, that may seem rather obvious when looking at the dictum only, but it is not obvious when one considers (the nature of) what is being indicated by 'it' - that is, a potential symptom of a psychiatric condition. Psychiatric conditions - and even more so symptoms that are a conceptual subset of ordinary experiences -are by nature notoriously varied, complex, and elusive of clearly defined boundaries [8]. That means a fallacy of equivocation may be embedded at this very place in the reasoning by the dictum 'understanding it makes it normal'. Consider for example how the fallacy may stem from simply substituting 'it' with a shift in generality between the two indications of 'it' as in 'Understanding emotions, makes sorrows normal'.

Embedded incongruence and resulting fallacies of equivocation may also arise in relation to various takes on the concept of normality in the reasoning expressed by the dictum 'understand-ing it makes it normal'. Again, considerations of the concept of mental illness vary greatly, including its (ab)normality in terms of statistics, epidemiology, prevalence, mortality, morbidity, social (un)acceptance, psychological strangeness, reproductive potential, actions, functions, harm potential, etc [8-11]. For example, some may take all illnesses categorically as being necessarily abnormal, in spite of some not being abnormal in all of the respects that I have just mentioned.

Similarly, embedded incongruence and resulting fallacies of equivocation may also arise in relation to various takes on 'understanding' in the reasoning by the dictum in question. Diagnostic understanding may entail approaches that vary greatly and are associated with different kinds of normality, when a matter of normality at all. 'Understanding' may refer to an understanding of the social cause of someone's condition as is seemingly the case in Meehl's work [2]. Perhaps contrary to Meehl's fallacy declaration, one may also legitimately talk of understanding the physiological causes of someone's condition. And various other kinds of under-standing have legitimate place in considerations of mental disorder including symptomatological, phenomenological, interpretative, psychodynamic, cognitive-behavioural, pragmatic, semantic and lexicological kinds of understanding [12]. 'Understanding' in relation to mental disorder also features in considerations of capacity, insight and acceptance [13-15].

Being clear about the embedded meaning in Meehl's work (and his dictum) may find congruence or expose a fallacy of equivocation, as just said. When considering the Gurova paper, being clear about the embedded meanings and their congruence is also important when taking issue with his work, for a mistaken equivocation may also arise in taking issue with another argument. In this regard, the Gurova paper seems to exceed Meehl's concept of normality to include also utilitarian aspects like the need for further care and abnormal consequences of a psychiatric condition. 
Thus, without being clear about the embedded meanings (as expressed through concepts, premises and logical inferences), we shall not be able to test whether diagnostic reasoning by the dictum 'understanding it makes it normal' is indeed fallacious. Gurova's paper contributes towards this clarity and has afforded opportunity here for the application and overview of some requirements for testing whether a particular sort of diagnostic reasoning is fallacious.

References

1. Gurova, L. (2013) Understanding it makes it normal: is it a reasoning fallacy or not? Journal of Evaluation in Clinical Practice, 19 (3), 524-527.

2. Meehl, P. (ed) (1973) Why I do not attend case conferences. In Psychodiagnosis: Selected Papers, pp. 225-302. Minneapolis, MN: University of Minnesota Press.

3. Hospers, J. (1973) An Introduction to Philosophical Analysis. pp. 128-138. London: Routledge \& Kegan Paul.

4. Martinich, A. P. (1996) Philosophical Writing, 2nd edn, pp. 18-35. Cambridge, MA: Blackwell.

5. Baggini, J. \& Fosl, P. S. (2003) The Philosopher's Toolkit. pp. 1-23. Oxford: Blackwell.

6. Van Staden, C. W. (2006) Conceptual issues in somatoform disorders and chronic fatigue syndrome. Current Opinion in Psychiatry, 18, 613-618.1

7. Kendell, R. \& Jablensky, A. (2003) Distinguishing between the validity and utility of psychiatric diagnoses. American Journal of Psychiatry, 160, 4-12.

8. Stein, D., Phillips, K. A., Bolton, D., Fulford, K. W. M., Sadler, J. Z. \& Kendler, K. S. (2010) What is a mental/psychiatric disorder? From DSM-IV to DSM-V. Psychological Medicine, 40, 17591765.

9. Bolton, D. (2008) What Is Mental Disorder. Oxford: Oxford University Press.

10. Fulford, K. W. M. (1989) Moral Theory and Medical Practice. Cam-bridge: Cambridge University Press.

11. Kendell, R. E. (1975) The concept of disease and its implication for psychiatry. British Journal of Psychiatry, 127, 305-315.

12. Van Staden, C. W. (2003) Diagnostic explanation and diagnostic understanding in psychiatry. Current Opinion in Psychiatry, 16, 667-672.

13. Van Staden, C. W. \& Krüger, C. (2003) Incapacity to give informed consent owing to mental disorder. Journal of Medical Ethics, 29, 41-43.

14. Van Staden, C. W. (2007) Can involuntarily admitted psychiatric patients give informed consent to participate in clinical research? South African Journal of Psychiatry, 13, 10-12.

15. Van Staden, C. W. (2009) Acceptance and insight: incapacity of give informed consent. Current Opinion in Psychiatry, 22, 554-558. 